\title{
Designing Unimodular Waveform with Low Range Sidelobes and Stopband for Cognitive Radar via Relaxed Alternating Projection
}

\author{
Xiang Feng, Yang-chun Song, Zhi-quan Zhou, and Yi-nan Zhao \\ School of Electronics and Information Engineering, Harbin Institute of Technology, No. 92 West Dazhi Street, \\ Nan Gang District, Harbin, Heilongjiang 150001, China
}

Correspondence should be addressed to Yi-nan Zhao; hrbzyn@163.com

Received 21 October 2015; Revised 1 February 2016; Accepted 1 March 2016

Academic Editor: Mourad Nedil

Copyright (C) 2016 Xiang Feng et al. This is an open access article distributed under the Creative Commons Attribution License, which permits unrestricted use, distribution, and reproduction in any medium, provided the original work is properly cited.

Cognitive radar could adapt the spectrum of waveforms in response to information regarding the changing environment, so as to avoid narrowband interference or electronic jamming. Besides stopband constraints, low range sidelobes and unimodular property are also desired. In this paper, we propose a Spectral Approximation Relaxed Alternating Projection (SARAP) method, to synthesize unimodular waveform with low range sidelobes and spectral power suppressed. This novel method, based on phase retrieval and relaxed alternating projection, could convert the correlation optimization into the spectrum approximation via the Fast Fourier Transform (FFT). Moreover, by virtue of the relaxation factor and accelerated factor, SARAP can exploit local area and more likely converge to the global solution. Numerical trials have demonstrated that SARAP could achieve excellent performance and computational efficiency which will facilitate the real-time design.

\section{Introduction}

The radar environment is nonstationary and often contaminated by electronic jamming or narrowband interference. Cognitive radar as a novel knowledge-aided mechanism could adapt the spectrum of waveforms to the changing environment $[1,2]$. Specifically, its emitted waveform should avert some frequency bands reserved for navigation or powerful emitters in the case of congested spectrum assignment, and spectral power in these frequency bands must be small enough [3-5]. Besides spectral requirement, low range sidelobes are also urgently required due to the influence on target detection. Moreover, another practical consideration is the constant envelope (i.e., the unimodular property) which permits efficient use of power amplifier in transmitter.

However, unimodular property maximizing transmitter's efficiency often leads waveform synthesis nonconvex. On the other hand, ideal sparse spectrum suppressing narrowband interference usually makes waveform with high range sidelobes [6]. In recent years, more and more researchers have paid attention to these issues. The new cyclic algorithm (CAN) and its improved versions (such as WeCAN, SCAN, and WeSCAN) have ever been introduced to design waveforms with low range sidelobes or sparse frequency properties $[6,7]$. Wang and Lu presented a novel cyclic mechanism combining the steepest descent algorithm, to compute sparse frequency waveform with sidelobes constraint [8]. Additionally, the authors in [9] proposed a majorization-minimization method to generate sparse frequency unimodular waveform with constraint on integrated sidelobe level (ISL). The LBFGS algorithm was also utilized to design unimodular waveform with low range sidelobes [10]. Other approaches, such as iterative method [11], alternating projection [12-14], and heuristic search algorithm [15], have also been presented. However, the initialization of these algorithms under unimodular constraint almost always incurs local stagnation and loss of robustness.

In this paper, we develop a Spectrum Approximation Relaxed Alternating Projection (SARAP) method based on phase retrieval and relaxed alternating projection, to generate unimodular waveform with low range sidelobes and sparse frequency properties. SARAP, incorporating the relaxation factor and accelerated factor (and based on FFT), could further enhance local exploiting and facilitate the real-time 
design. The rest of our paper is organized as follows. Section 2 describes the signal model and problem statement. Section 3 develops several different SARAP frameworks. Section 4 provides series of numerical simulations. Finally, Section 5 concludes the paper.

\section{Signal Model and Problem Statement}

In this section, we aim at several typical waveform requirements and assume that the emitted waveform is phasemodulated. So the phase-modulated waveform which possesses constant modulus property is preferable for consistent maximum power transmission $[8,16]$. Let $\mathbf{x} \in \mathbb{C}^{N \times 1}$ denote the discrete waveform vector; it could be cast as

$$
\mathbf{x}=\left[\begin{array}{llll}
e^{j \psi_{1}} & e^{j \psi_{2}} & \cdots & e^{j \psi_{N}}
\end{array}\right]^{T}
$$

where $\psi_{n}$ denotes the phase of the $n$th element, $(\cdot)^{T}$ indicates the matrix or vector transpose, $\mathbb{C}$ represents the complex field, and $N$ is the number of samples. As discussed in [13, $14,17]$, the correlation vector $\boldsymbol{\alpha}(\mathbf{x})$ could be regarded as the output of matched filter, and each entry of $\boldsymbol{\alpha}(\mathbf{x})$ has $\alpha_{n}(\mathbf{x})=$ $\sum_{k=1}^{N-n} \mathbf{x}(k) \mathbf{x}(k+n)^{*}=\alpha_{-n}^{*}(\mathbf{x})$, and $(\cdot)^{*}$ denotes the complex conjugate. Since even-number (e.g., $2 N$ ) is more suitable for implementing FFT, we usually pad " 0 " to the tail of $\boldsymbol{\alpha}(\mathbf{x})$ to make $\boldsymbol{\alpha}(\mathbf{x}) \in \mathbb{C}^{2 N \times 1}$ for the convenience of FFT.

Note that low range sidelobes of waveform could improve target detection when range compression is applied in receiver [12-14]. Generally, low range sidelobes could be obtained by minimizing the ISL $[6,8]$ as follows:

$$
\min _{\mathbf{x}} \mathrm{ISL}=\sum_{n=-(N-1)}^{N-1}\left|\alpha_{n}(\mathbf{x})\right|^{2}-\alpha_{0}(\mathbf{x})
$$

subject to: $\quad|\mathbf{x}(n)|=1, \quad n=1,2, \ldots, N$,

and the ISL metric in (2), via the Parseval-type equality [18], could be expressed as

$$
\sum_{n=-(N-1)}^{N-1}\left|\alpha_{n}(\mathbf{x})-N \delta_{n}\right|^{2}=\frac{1}{2 N} \sum_{p=1}^{2 N}\left|\boldsymbol{\rho}\left(\omega_{p}\right)-N\right|^{2}
$$

where $\delta_{n}=1$ only if $n=0$, and else $\delta_{n}=0$ only if $n \neq 0 ; \omega_{p}=(2 \pi / 2 N) p,(p=1,2, \ldots, 2 N)$, and $\rho\left(\omega_{p}\right)=$ $\left|\sum_{n=1}^{N} \mathbf{x}(n) e^{-j \omega_{p} n}\right|^{2}$. Based on discussion in [6, 7], an equivalent form of (3) with lower order is "almost equivalent" to the one as follows:

$$
\min _{\mathbf{x}} \frac{1}{2 N} \sum_{p=1}^{2 N} \|\left|\sum_{n=1}^{N} \mathbf{x}(n) e^{-j \omega_{p} n}\right|-\left.\sqrt{N}\right|^{2}
$$

subject to: $\quad|\mathbf{x}(n)|=1, \quad n=1,2, \ldots, N$.

Making full use of the $2 \mathrm{~N} \times 2 \mathrm{~N}$ unitary DFT matrix $\mathbf{F}_{2 N}$, that is, $\mathbf{F}_{2 N}^{H} \mathbf{F}_{2 N}=\mathbf{I}$, where $\mathbf{I}$ denotes the unitary matrix, the objective function (4) could expand to another equivalent form by enforcing the Euclidean norm requirement. Thus range sidelobes could be suppressed by solving following problem:

$$
\min _{\mathbf{x}} J_{1}(\mathbf{x})=\left\|\mathbf{F}_{2 N}\left[\begin{array}{c}
\mathbf{x} \\
\mathbf{0}_{N \times 1}
\end{array}\right]-\frac{1}{\sqrt{2}} \cdot \mathbf{v}\right\|^{2}
$$

subject to: $\quad|\mathbf{x}(n)|=1, \quad n=1,2, \ldots, N$

$$
\mathbf{v}=\left[\begin{array}{llll}
e^{j \varphi_{1}} & e^{j \varphi_{2}} & \cdots & e^{j \varphi_{2 N}}
\end{array}\right]
$$

where $(\cdot)^{H}$ means the conjugate transpose, $\|\cdot\|$ denotes the Euclidean norm, $\mathbf{0}_{N \times 1}$ denotes all-zero vector with size $N \times 1$, and $\varphi_{n}$ represents the auxiliary phase. As a consequence, the ISL minimizing in (2) could be expressed as the spectrum approximation of (5), and auxiliary phase $\varphi_{n}$ could be achieved by the FFT-phase of $\mathbf{x}$.

In another scenario, some powerful dot-scatters existing in the $q$ th range interval would inevitably affect target detection in the $r$ th interval due to high range sidelobes of waveform. We assume that the velocity of scatters or targets is so small that the Doppler frequency shift could be negligible. Detailed information about this machinery, of which high range sidelobes impacts on target detection, could refer to [14]. In some cases, low range sidelobes in prescribed intervals would make a difference for detecting targets. Accordingly, whole suppression under ISL metric seems unnecessary. Only prescribed intervals $Q_{r}$ need to be suppressed which could be obtained by some prior information and these intervals can be expressed as

$$
Q_{r}=\left\{ \pm|z-q|: z \in \widehat{Z}_{r}\right\} \backslash\{N-1,-N+1\}
$$

where $\widehat{Z}_{r}$ denotes the union of all suspicious or foreseeable intervals with interesting targets, and let vector $\mathbf{z}=$ $\left[\mathbf{z}_{1}, \mathbf{z}_{2}, \ldots, \mathbf{z}_{n}, \ldots \mathbf{z}_{2 N}\right]^{T}$ be the indicating vector:

$$
\mathbf{z}_{n}= \begin{cases}0, & n \in Q_{r} \\ 1, & n \notin Q_{r}\end{cases}
$$

Suppose that $\widetilde{\mathbf{x}}$ represents the desired waveform (i.e., the ideal waveform) with $\alpha_{n}(\widetilde{\mathbf{x}})=0$ only if $n \in Q_{r}$, and here $\boldsymbol{\alpha}(\widetilde{\mathbf{x}})=\operatorname{Diag}(\mathbf{z}) \cdot \boldsymbol{\alpha}(\mathbf{x})$; namely, $\alpha_{n}(\widetilde{\mathbf{x}})=\alpha_{n}(\mathbf{x})$ if $n \notin Q_{r}$, where $\operatorname{Diag}(\mathbf{z})$ denotes a diagonal matrix formed by elements of $\mathbf{z}$. The designed waveform $\mathbf{x}$, attempting to satisfy the ideal requirement, should enforce its correlation vector approximating to the one of $\widetilde{\mathbf{x}}$. Therefore, this approximating mechanism could be formulated as

$$
\begin{array}{cl}
\min _{\mathbf{x}} & \|\boldsymbol{\alpha}(\mathbf{x})-\boldsymbol{\alpha}(\widetilde{\mathbf{x}})\|^{2} \\
\text { subject to: } & |\mathbf{x}(n)|=1, \quad n=1,2, \ldots, N .
\end{array}
$$

With Parseval-type equality taken into account, that is, $\left\|\mathbf{F}_{2 N} \mathbf{x}\right\|^{2}=\|\mathbf{x}\|^{2}[18]$, the objective function (8) could be equivalently rewritten as

$$
\min _{\mathbf{x}} J_{2}(\mathbf{x})=\left\|\mathbf{F}_{2 N} \boldsymbol{\alpha}(\widetilde{\mathbf{x}})-\mathbf{F}_{2 N} \boldsymbol{\alpha}(\mathbf{x})\right\|^{2}
$$

subject to: $\quad|\mathbf{x}(n)|=1, \quad n=1,2, \ldots, N$, 
where $\mathbf{F}_{2 N} \boldsymbol{\alpha}(\widetilde{\mathbf{x}})=\widetilde{\mathbf{f}} \odot \widetilde{\mathbf{f}}^{*}$ represents PSD of desired waveform, $\widetilde{\mathbf{f}}=\left(\mathbf{F}_{2 N} \boldsymbol{\alpha}(\widetilde{\mathbf{x}})\right)^{1 / 2} \odot \mathbf{u}$ denotes the desired frequency spectrum, $\odot$ means the element-wise Hadamard product, $(\cdot)^{1 / 2}$ indicates the element-wise square root, and $\mathbf{u}=\left[\begin{array}{llll}e^{j \phi_{1}} & e^{j \phi_{2}} & \cdots & e^{j \phi_{2 N}}\end{array}\right]^{T}$ represents the auxiliary vector. Let $\mathbf{C}=\left[\begin{array}{ll}\mathbf{I} & \mathbf{0}\end{array}\right]$ denote the extended or truncated matrix; there would be $\mathbf{F}_{2 N} \boldsymbol{\alpha}(\mathbf{x})=$ $\left(\mathbf{F}_{2 N} \mathbf{C}^{T} \mathbf{x}\right) \odot\left(\mathbf{F}_{2 N} \mathbf{C}^{T} \mathbf{x}\right)^{*}$. The objective function (9), via the "almost equivalence" $[6,7]$, could be expressed as

$$
\begin{array}{cl}
\min _{\mathbf{x}} & J_{2}(\mathbf{x})=\left\|\left(\mathbf{F}_{2 N} \boldsymbol{\alpha}(\widetilde{\mathbf{x}})\right)^{1 / 2} \odot \mathbf{u}-\mathbf{F}_{2 N} \mathbf{C}^{T} \mathbf{x}\right\|^{2} \\
\text { subject to: } & |\mathbf{x}(n)|=1, \quad n=1,2, \ldots, N
\end{array}
$$

so the objective function (8) corresponding to the correlation approximating could be expressed as the spectrum approximation of (10). One solution without constraint on unimodular property could be obtained as $\overline{\mathbf{x}}=\mathbf{C}^{T} \mathbf{F}_{2 N}^{H}\left(\left(\mathbf{F}_{2 N} \boldsymbol{\alpha}(\widetilde{\mathbf{x}})\right)^{1 / 2} \odot\right.$ $\mathbf{u}$ ), where vector $\mathbf{u}$ corresponds to the FFT-phase of $\mathbf{x}$, that is, $\mathbf{u}=\exp \left(j \phi\left(\mathbf{F}_{2 N} \mathbf{C}^{T} \mathbf{x}\right)\right), \exp (\cdot)$ denotes the element-wise exponential function, and $\phi(\cdot)$ represents the element-wise phase extraction. Finally, the designed waveform $\mathbf{x}$ would be determined via the phase information of $\overline{\mathbf{x}}$.

Additionally, the emitted waveform should avert some frequency bands reserved for navigation or military communications. More realistic to say, spectral power in these frequency bands must be small enough; suppose that the suppressed frequency bands (i.e., stopbands) could be expressed as

$$
\begin{aligned}
\Omega= & \left\{\left(f_{11}, f_{12}\right),\left(f_{21}, f_{22}\right), \ldots,\left(f_{k 1}, f_{k 2}\right), \ldots,\right. \\
& \left.\left(f_{N_{s} 1}, f_{N_{s} 2}\right)\right\}
\end{aligned}
$$

where $f_{k 1}$ and $f_{k 2}$ represent the lower and upper frequencies, respectively, for the $k$ th stopband, and $N_{s}$ is the number of these stopbands. As is known the Fourier transform of $\mathbf{x}$ could be written as $\mathbf{F}_{2 N} \mathbf{C}^{T} \mathbf{x}$. We extract a matrix $\mathbf{P}$ from rows of $\mathbf{F}_{2 N}$ corresponding to $\Omega$ and then utilize vector $\widetilde{\mathbf{w}}$ to indicate this corresponding relation; namely, $\widetilde{\mathbf{w}}_{k}=1$ only if $f_{k} \in \Omega$ and $\widetilde{\mathbf{w}}_{k}=0$ when $f_{k} \notin \Omega$, where $f_{k}$ represents the discrete frequency sample. Therefore, matrix $(\mathbf{I}-\operatorname{Diag}(\widetilde{\mathbf{w}})) \mathbf{F}_{2 N}$ would represent the remaining rows of $\mathbf{F}_{2 N}$. Based on discussion above, spectral power in stopbands could be expressed as $\min _{\mathbf{x}}\left\|\mathbf{P C} \mathbf{C}^{T} \mathbf{x}\right\|^{2}$. Referring to [13], this objective function could be further rewritten as follows:

$$
\begin{aligned}
\min _{\mathbf{x}} & J_{3}(\mathbf{x}) \\
& =\left\|\mathbf{F}_{2 N} \mathbf{C}^{T} \widetilde{\mathbf{x}}-(\mathbf{I}-\operatorname{Diag}(\widetilde{\mathbf{w}})) \mathbf{F}_{2 N} \mathbf{C}^{T} \mathbf{x}\right\|^{2}
\end{aligned}
$$

subject to: $\quad|\mathbf{x}(n)|=1, \quad n=1,2, \ldots, N$;

thus the spectral power suppression could be formulated as the spectrum approximation, and one solution without constraint on unimodular property is $\widehat{\overline{\mathbf{x}}}=\mathbf{C}^{T} \mathbf{F}_{2 N}^{H}[(\mathbf{I}-$ $\left.\operatorname{Diag}(\widetilde{\mathbf{w}}))\left(\mathbf{F}_{2 N} \mathbf{C}^{T} \mathbf{x}\right)\right]$; then the designed waveform $\mathbf{x}$ could be determined via the phase information of $\widehat{\overline{\mathbf{x}}}$.

\section{Waveform Synthesis via SARAP}

In this section, three typical waveform tasks for cognitive radar scenario have been listed as follows:

(a) Design unimodular waveform with low range sidelobes in prescribed intervals.

(b) Design unimodular waveform with ISL suppressed and sparse frequency properties.

(c) Design unimodular waveform with low range sidelobes in prescribed intervals and sparse frequency properties as well.

Task (a) attempts to design unimodular waveform with low range sidelobes in prescribed intervals; thus the objective function (9) under unimodular constraint could be expressed as

$$
\begin{aligned}
\min _{\mathbf{x}} & J_{a}(\mathbf{x})=\left\|\mathbf{F}_{2 N} \boldsymbol{\alpha}(\widetilde{\mathbf{x}})-\mathbf{F}_{2 N} \boldsymbol{\alpha}(\mathbf{x})\right\|^{2} \\
\text { subject to: } & |\mathbf{x}(n)|=1, \quad n=1,2, \ldots, N .
\end{aligned}
$$

Task (b) means that low range sidelobes and sparse frequency properties should be considered together. In order to design unimodular waveform under this case, we select $\gamma \in(0,1)$ to balance ISL performance and sparse frequency property and finally formulate the optimization problem as follows:

$$
\begin{aligned}
\min _{\mathbf{x}} & J_{b}(\mathbf{x})=(1-\gamma) \cdot J_{1}(\mathbf{x})+\gamma \cdot J_{3}(\mathbf{x}) \\
\text { subject to: } & |\mathbf{x}(n)|=1, \quad n=1,2, \ldots, N .
\end{aligned}
$$

Task (c) means that suppressing powerful interference in prescribed range intervals seems requisite; meanwhile sparse frequency property should also be considered. To accomplish this task, we select $\gamma \in(0,1)$ to balance the range sidelobes and sparse frequency property and then formulate the optimization problem under unimodular constraint, as follows:

$$
\begin{aligned}
\min _{\mathbf{x}} & J_{c}(\mathbf{x})=(1-\gamma) \cdot J_{2}(\mathbf{x})+\gamma \cdot J_{3}(\mathbf{x}) \\
\text { subject to: } & |\mathbf{x}(n)|=1, \quad n=1,2, \ldots, N .
\end{aligned}
$$

This constrained optimization could be regarded as phase retrieval, and phase retrieval under unimodular constraint is generally nonconvex [19]. Solving these problems efficiently is challengeable because of its nondeterministic polynomialtime hard property. Alternating projection (AP) method as a cyclic mechanism was introduced for these problems $[20,21]$, and our previous works have also demonstrated its performance $[13,14]$. Particularly, let $A$ denote the collection of waveforms which possesses constant envelope, that is, $|\mathbf{x}(n)|=1, n=1,2, \ldots, N$, and let $B$ denote the collection which satisfies objective functions above; thus AP is to seek element $\bar{x}$ of $A$ which has the nearest distance to element $y$ of $B$ and then run round and round. Define $\bar{x}=\operatorname{Proj}_{A}(y)=$ $\arg \min _{x \in A}\|x-y\|$ as the projection-entry; in the same way, we could obtain $\bar{y}=\operatorname{Proj}_{B}(\widehat{x})=\arg \min _{y \in B}\|\bar{x}-y\|$. Namely, the 
intuition behind AP is just as mapping $T: A \rightarrow B \rightarrow A$; that is, $x^{(k+1)}=T \cdot x^{(k)}=\operatorname{Proj}_{A}\left(\operatorname{Proj}_{B}\left(x^{(k)}\right)\right)$, where $x^{(k)}$ denotes the solution of the $k$ th iteration. Consider that AP generates a sequence of iterations, $\left\{\left(x^{(k)}, y^{(k)}\right)\right\}$ per iteration; that is, $\left\|x^{(k+1)}-y^{(k+1)}\right\| \leq\left\|x^{(k)}-y^{(k+1)}\right\| \leq\left\|x^{(k)}, y^{(k)}\right\|$ which has the asymptotic convergence. As different initialization of algorithms, under the nonconvex case, would incur different convergence. The authors in [22] held that AP among two convex intersecting sets could not always be converged in the sense of norm, which means that this convergence might be undetermined. In order to make convergence powerful and further reduce the execution time, relaxed alternating projection (RAP) could be feasible. The authors in $[23,24]$ presented RAP to tackle the constrained optimization and found that it can surely expedite converging. To describe main procedures of RAP, let $\lambda \in(0,1]$ and $\delta(\cdot)$ represent relaxation factor and accelerated factor, respectively; then RAP could be formulated as

$$
\begin{aligned}
& T_{\delta(\cdot), \lambda} x \\
& \quad=\operatorname{Proj}_{A}\left(x+\lambda \cdot \delta(x) \cdot\left(\operatorname{Proj}_{A}\left(\operatorname{Proj}_{B}(x)\right)-x\right)\right),
\end{aligned}
$$

where $\delta(x)$ has several forms as follows:

(i) $\delta(x)=\left\|\operatorname{proj}_{B}(x)-x\right\|^{2} /\left\|\operatorname{proj}_{A}\left(\operatorname{proj}_{B}(x)\right)-x\right\|^{2}$,

(ii) $\delta(x)=1+\| \operatorname{proj}_{A}\left(\operatorname{proj}_{B}(x)\right)-$ $\operatorname{proj}_{B}(x)\left\|^{2} /\right\| \operatorname{proj}_{A}\left(\operatorname{proj}_{B}(x)\right)-x \|^{2}$,

(iii) $\delta(x)=\left\langle x, x-\operatorname{proj}_{A}\left(\operatorname{proj}_{B}(x)\right)\right\rangle / \| \operatorname{proj}_{A}\left(\operatorname{proj}_{B}(x)\right)-$ $x \|^{2}$.

$\langle\cdot, \cdot\rangle$ denotes the scalar product, and our simulations mainly adopt (ii). RAP could exploit consecutive projections via its relaxation factor and accelerated factor. As shown in equations (i), (ii) and (iii), both $\left(\operatorname{proj}_{A}\left(\operatorname{proj}_{B}(x)\right)-\operatorname{proj}_{B}(x)\right)$ and $\left(\operatorname{Proj}_{A}\left(\operatorname{Proj}_{B}(x)\right)-x\right)$ represent the pertinence-difference in different projection collections. These differences could assist RAP to get rid of local stagnation. Additionally, the relaxation factor and accelerated factor could mutually reduce the number of iterations (i.e., computational expense) required for converging; meanwhile, this mechanism has also kept the progressive convergence $[23,24]$.

Next, we would introduce SARAP to solve tasks (a), (b), and (c). Considering that cyclic methods or iterative methods only with large number of iterations could approximate to the optimal solution which seems impractical for engineering, here we adopt a conventional way to evaluate the performance of algorithms $[6,7,13,14]$. For instance, define the total number of iterations or norm-error of solutions as the stopping criterion; namely, let $K$ denote the total number and let $\varepsilon$ signify the predefined norm-error threshold.

Algorithm 1. SARAP for designing waveform with low range sidelobes in prescribed intervals:

(1) Initialize $\mathbf{x}^{(0)}$ with the random sequence or some given sequence (e.g., the Frank sequence), and select $\lambda \in(0,1]$.

(2) Depending on the latest value of $\mathbf{x}^{(k)}$, compute $\mathbf{f}^{(k)}$ using $\mathbf{f}^{(k)}=\left(\mathbf{F}_{2 N} \boldsymbol{\alpha}(\widetilde{\mathbf{x}})\right)^{1 / 2} \odot \exp \left(j \phi\left(\mathbf{F}_{2 N} \mathbf{C}^{T} \mathbf{x}^{(k)}\right)\right)$.
(3) Depending on the latest value of $\mathbf{f}^{(k)}$, compute $\widehat{\mathbf{x}}^{(k)}=$ $\exp \left(j \phi\left(\mathbf{C F}_{2 N}^{H} \mathbf{f}^{(k)}\right)\right)$.

(4) Use RAP to obtain the improved solution: $\mathbf{x}^{(k+1)}=$ $\exp \left(j \phi\left(\mathbf{x}^{(k)}+\lambda \cdot \delta\left(\mathbf{x}^{(k)}\right) \cdot\left(\widehat{\mathbf{x}}^{(k)}-\mathbf{x}^{(k)}\right)\right)\right)$.

(5) Repeat steps (2)-(4) until the stopping criterion is satisfied; for example, $k>K$ or $\left\|\mathbf{x}^{(k)}-\mathbf{x}^{(k+1)}\right\|<\varepsilon$.

Algorithm 2. SARAP for designing unimodular waveform with ISL suppressed and sparse frequency properties:

(1) Initialize $\mathbf{x}^{(0)}$ with the random sequence or some given sequence (e.g., the Frank sequence), and select $\lambda \in(0,1]$.

(2) Depending on the latest value of $\mathbf{x}^{(k)}$, compute $\mathbf{f}_{B_{1}}^{(k)}$ and $\mathbf{f}_{B_{3}}^{(k)}$ using $\mathbf{f}_{B_{1}}^{(k)}=1 / \sqrt{2} \cdot \exp \left(j \phi\left(\mathbf{F}_{2 N} \mathbf{C}^{T} \mathbf{x}^{(k)}\right)\right)$ and $\mathbf{f}_{B_{3}}^{(k)}=$ $(\mathbf{I}-\operatorname{Diag}(\widetilde{\mathbf{w}}))\left(\mathbf{F}_{2 N} \mathbf{C}^{T} \mathbf{x}^{(k)}\right)$.

(3) Depending on the latest values of $\mathbf{f}_{B_{1}}^{(k)}$ and $\mathbf{f}_{B_{3}}^{(k)}$, compute $\widehat{\mathbf{x}}^{(k)}=\exp \left(j \phi\left((1-\gamma) \cdot\left(\mathbf{C F}_{2 N}^{H} \mathbf{f}_{B_{1}}^{(k)}\right)+\gamma \cdot\left(\mathbf{C F}_{2 N}^{H} \mathbf{f}_{B_{3}}^{(k)}\right)\right)\right)$.

(4) Use RAP to obtain the improved solution: $\mathbf{x}^{(k+1)}=$ $\exp \left(j \phi\left(\mathbf{x}^{(k)}+\lambda \cdot \delta\left(\mathbf{x}^{(k)}\right) \cdot\left(\widehat{\mathbf{x}}^{(k)}-\mathbf{x}^{(k)}\right)\right)\right)$.

(5) Repeat step (2)-(4) until the stopping criterion is satisfied; for example, $k>K$ or $\left\|\mathbf{x}^{(k)}-\mathbf{x}^{(k+1)}\right\|<\varepsilon$.

Algorithm 3. SARAP for designing unimodular waveform with low range sidelobes in prescribed intervals and sparse frequency properties as well:

(1) Initialize $\mathbf{x}^{(0)}$ with the random sequence or some given sequence (e.g., the Frank sequence), and select $\lambda \in(0,1]$.

(2) Depending on the latest value $\mathbf{x}^{(k)}$, compute $\mathbf{f}_{B_{2}}^{(k)}$ and $\mathbf{f}_{B_{3}}^{(k)}$ using $\mathbf{f}_{B_{2}}^{(k)}=\left(\mathbf{F}_{2 N} \boldsymbol{\alpha}(\widetilde{\mathbf{x}})\right)^{1 / 2} \odot \exp \left(j \boldsymbol{\phi}\left(\mathbf{F}_{2 N} \mathbf{C}^{T} \mathbf{x}^{(k)}\right)\right)$ and $\mathbf{f}_{B_{3}}^{(k)}=(\mathbf{I}-\operatorname{Diag}(\widetilde{\mathbf{w}}))\left(\mathbf{F}_{2 N} \mathbf{C}^{T} \mathbf{x}^{(k)}\right)$.

(3) Depending on the latest values of $\mathbf{f}_{B_{2}}^{(k)}$ and $\mathbf{f}_{B_{3}}^{(k)}$, compute $\widehat{\mathbf{x}}^{(k)}=\exp \left(j \phi\left((1-\gamma) \cdot\left(\mathbf{C F}_{2 N}^{H} \mathbf{f}_{B_{2}}^{(k)}\right)+\gamma \cdot\left(\mathbf{C F}_{2 N}^{H} \mathbf{f}_{B_{3}}^{(k)}\right)\right)\right)$.

(4) Use RAP to obtain the improved solution: $\mathbf{x}^{(k+1)}=$ $\exp \left(j \phi\left(\mathbf{x}^{(k)}+\lambda \cdot \delta\left(\mathbf{x}^{(k)}\right) \cdot\left(\widehat{\mathbf{x}}^{(k)}-\mathbf{x}^{(k)}\right)\right)\right)$.

(5) Repeat steps (2)-(4) until the stopping criterion is satisfied; for example, $k>K$ or $\left\|\mathbf{x}^{(k)}-\mathbf{x}^{(k+1)}\right\|<\varepsilon$.

\section{Numerical Examples}

4.1. Designing Unimodular Waveform with Low Range Sidelobes in Prescribed Intervals. Suppose that some interference locates in range intervals $Q_{r_{1}}=[1: 40]$ or $Q_{r_{2}}=[20: 40] \mathrm{U}$ $[50: 70]$ and their velocity is so small that the Doppler frequency shift could be negligible. Additionally, the pulse duration of waveform is $200 \mu \mathrm{s}$ and the sampling rate is $980 \mathrm{KHz}$; thus the number of samples is $N=196$. Meanwhile a novel 


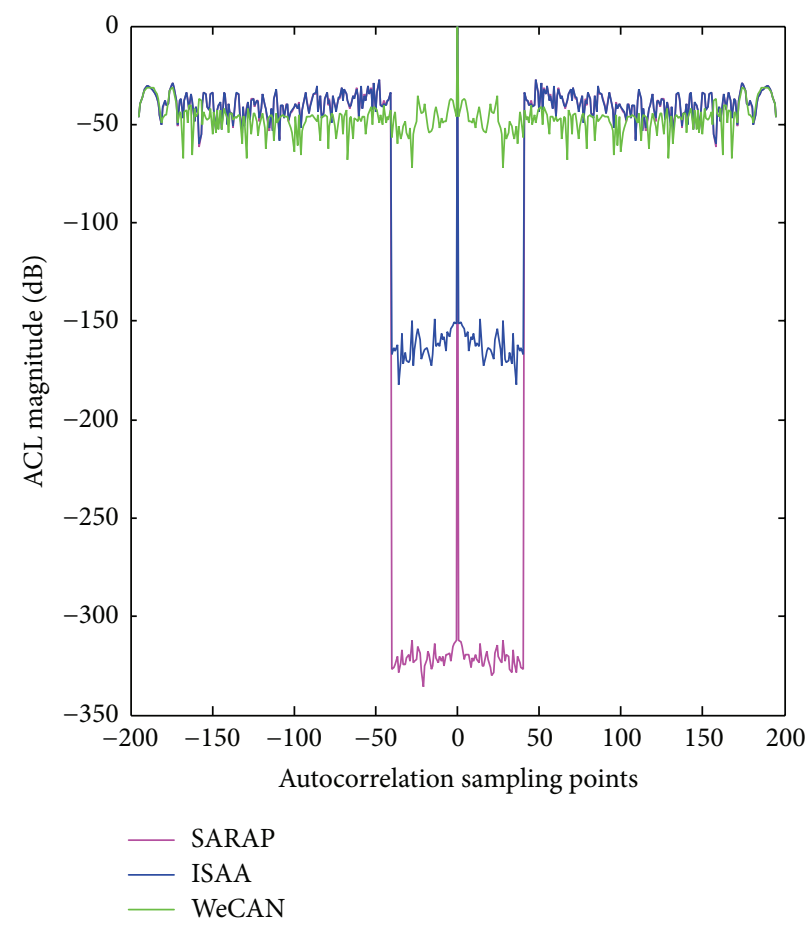

(a)

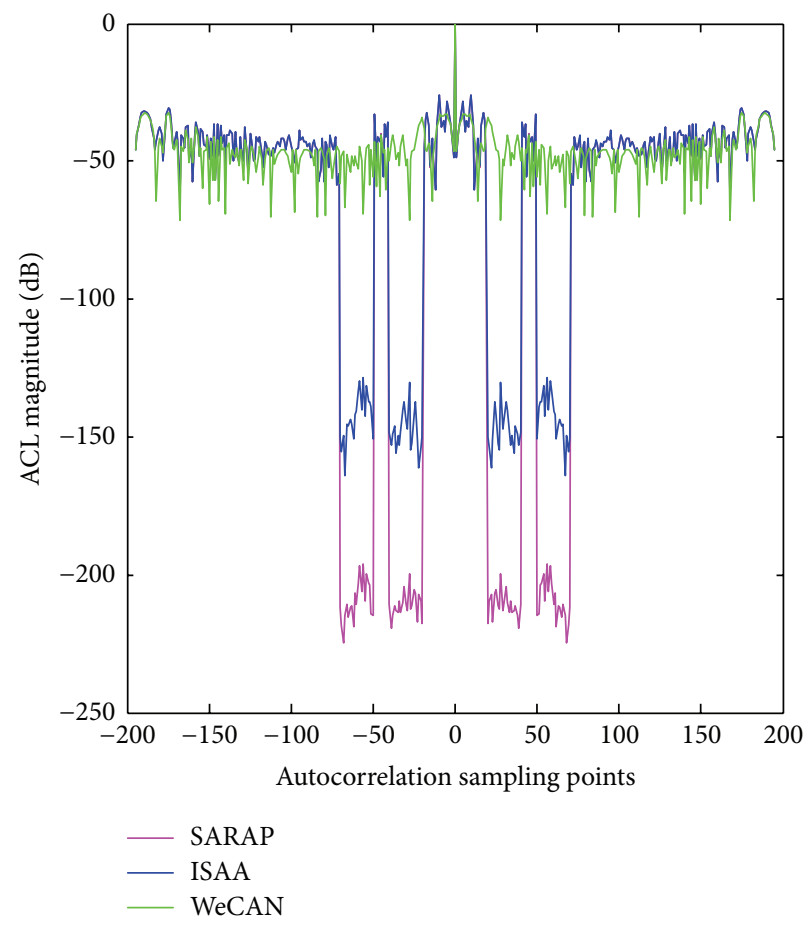

(b)

FIGURE 1: ACL comparison for suppressing sidelobes; (a) illustrates the case $Q_{r_{1}}=[1: 40]$; (b) illustrates the case $Q_{r_{2}}=[20: 40] \cup[50: 70]$.

performance metric, that is, autocorrelation level (ACL), is given by

$$
\operatorname{ACL}(n)=20 \log \frac{\left|\alpha_{n}(\mathbf{x})\right|}{\left|\alpha_{0}(\mathbf{x})\right|}(\mathrm{dB}) \quad n=1,2, \ldots, N-1 .
$$

Moreover, let Aver-ACL denote the averaged ACL in suppressed intervals. As engineering usually cares about execution time, here let $K=1000$ and $\varepsilon=10^{-14}$ signify the predefined termination. Then SARAP in Algorithm 1 would be compared with WeCAN [6] and ISAA [14]. Note that the initialization of algorithms is indeed significant for cyclic algorithm or alternating projection. Firstly, these algorithms would be initialized by the Frank sequence. For the length $N=M^{2}$, the Frank sequence is given by

$$
\mathbf{x}(n M+k+1)=e^{j 2 \pi n k / M}, \quad n, k=0,1, \ldots, M-1 .
$$

Here, we must state that our simulations perform on PC with $3.40 \mathrm{GHz}$ i7 $4770 \mathrm{CPU}$ and $8 \mathrm{G}$ RAM.

One could easily find that, from Table 1 , the case $Q_{r_{2}}=$ $[20: 40] \cup[50: 70]$ has consumed more time than that in $Q_{r_{1}}$ under identical parameters; that is, $K=1000$ and $\varepsilon=10^{-14}$. ISAA and SARAP consume less time than WeCAN, and WeCAN has lost its superiority in terms of execution time and Aver-ACL when compared with others. Although the time difference of SARAP and ISAA seems slight, Aver-ACL could make a difference. From Figure 1, all algorithms initialized by the Frank sequence have shown some distinguishable results, which means that SARAP has achieved the best Aver-ACL in both $Q_{r_{1}}$ and $Q_{r_{2}}$, and WeCAN under same condition has
TABLE 1: Algorithms (initialized by Frank sequence) comparison for

\begin{tabular}{|c|c|c|c|c|}
\hline \multirow[b]{2}{*}{ Algorithms } & \multicolumn{2}{|c|}{$Q_{r_{1}}=[1: 40]$} & \multicolumn{2}{|c|}{$Q_{r_{2}}=[20: 40] \cup[50: 70]$} \\
\hline & $\begin{array}{c}\text { Execution } \\
\text { time (s) }\end{array}$ & $\begin{array}{l}\text { Aver-ACL } \\
\quad(\mathrm{dB})\end{array}$ & $\begin{array}{c}\text { Execution } \\
\text { time }(\mathrm{s})\end{array}$ & $\begin{array}{l}\text { Aver-ACL } \\
\quad(\mathrm{dB})\end{array}$ \\
\hline SARAP & 0.086 & -321.460 & 0.122 & -210.308 \\
\hline ISAA & 0.173 & -162.577 & 0.197 & -145.383 \\
\hline WeCAN & 5.241 & -48.839 & 5.431 & -45.466 \\
\hline
\end{tabular}
suppressing range sidelobes.

lost its superiority. This phenomenon in Figure 1 and Table 1 attributes that SARAP could expedite converging and more likely approximate to the global solution, while ISAA and WeCAN with same parameters might suffer local stagnation.

Additionally, algorithms initialized by random sequence would be considered. Here, we adopt 100 independent trials to evaluate their performance, let AVER-time denote the averaged execution time, and let AVER-ACL represent the averaged ACL in prescribed intervals for 100 trials. At the beginning of each trial, all algorithms should be initialized by identical random sequence.

SARAP, in Figure 2 and Table 2, has achieved the best AVER-ACL for both $Q_{r_{1}}$ and $Q_{r_{2}}$, while WeCAN might lose its superiority. Meanwhile, one phenomenon could be observed that all algorithms for $Q_{r_{2}}$ have occupied more time than $Q_{r_{1}}$, ISAA and SARAP have consumed less time than WeCAN. Although the time difference between SARAP and ISAA seems slight, the AVER-ACL comparison would make a difference. Compared with Figure 1 and Table 1, random 


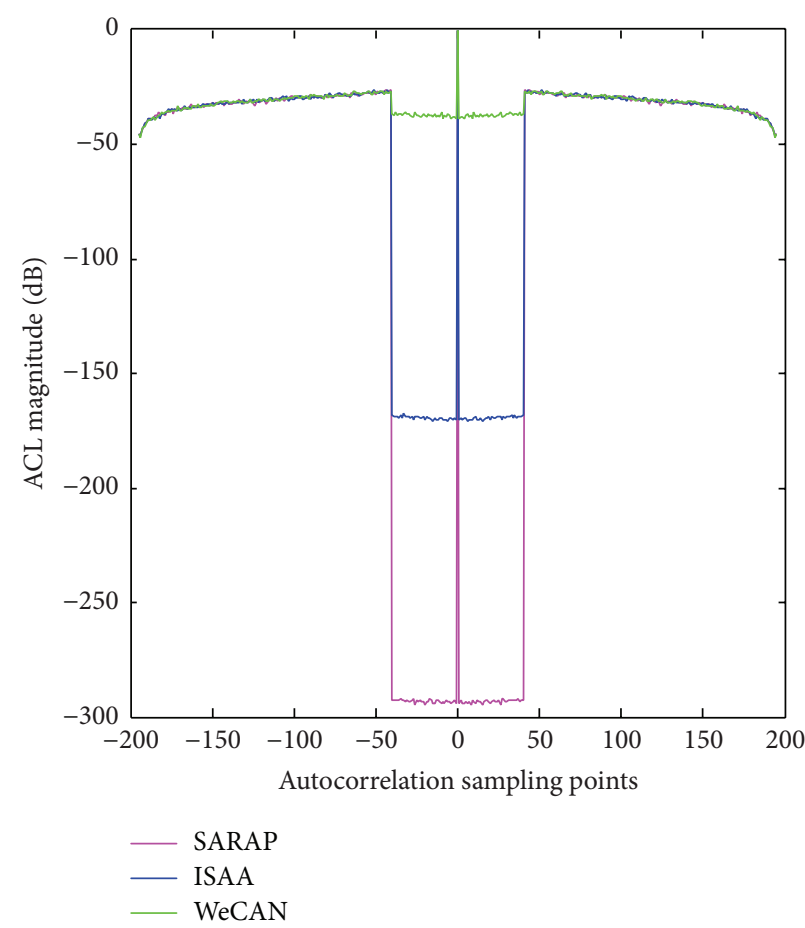

(a)

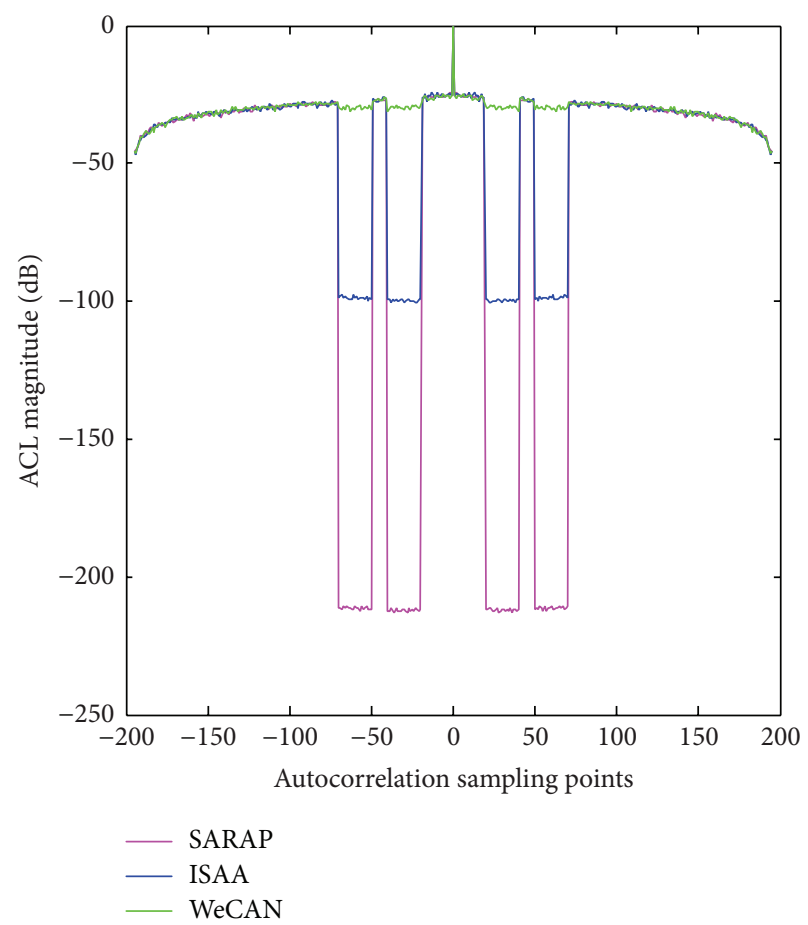

(b)

FIGURE 2: ACL comparison for suppressing sidelobes; (a) illustrates the case $Q_{r_{1}}=[1: 40]$; (b) illustrates the case $Q_{r_{2}}=[20: 40] \cup[50: 70]$.

TABLE 2: Algorithms (initialized by random sequence) comparison for suppressing range sidelobes.

\begin{tabular}{lcccc}
\hline Algorithms & \multicolumn{2}{c}{$Q_{r_{1}}=[1: 40]$} & \multicolumn{2}{c}{$Q_{r_{2}}=[20: 40] \cup[50: 70]$} \\
& $\begin{array}{c}\text { AVER-time } \\
(\mathrm{s})\end{array}$ & $\begin{array}{c}\text { AVER-ACL } \\
(\mathrm{dB})\end{array}$ & $\begin{array}{c}\text { AVER-time } \\
(\mathrm{s})\end{array}$ & $\begin{array}{c}\text { AVER-ACL } \\
(\mathrm{dB})\end{array}$ \\
\hline SARAP & 0.143 & -293.167 & 0.165 & -211.702 \\
ISAA & 0.161 & -169.437 & 0.182 & -99.278 \\
WeCAN & 4.952 & -32.127 & 5.553 & -28.030 \\
\hline
\end{tabular}

initialization would make some influence on the robustness of algorithms, and the averaging performance comparison for 100 independent trials could signify this robustness which is beneficial for engineering.

4.2. Designing Unimodular Waveform with ISL Suppressed and Sparse Frequency Properties. Based on radar scenario in [25], we suppose that multiple stopbands locate in $[4.2,4.3] \mathrm{U}$ $[4.5,4.6] \mathrm{MHz}$, and passbands would cover the remaining part of $[4.0,5.0] \mathrm{MHz}$. Additionally, the pulse duration is 400 $\mu$ s and sampling rate is $1 \mathrm{MHz}$; then there are 400 samples. SARAP of Algorithm 2 would be compared with WeSCAN and SCAN [7] under parameters $\gamma=0.6, K=1000$, and $\varepsilon=10^{-8}$. Furthermore, all algorithms would be initialized by identical random sequence. Autocorrelation sidelobe peak (ASP) and peak stopband power (PSP) are given by

$$
\begin{aligned}
\mathrm{ASP}=20 \log \left(\max \frac{\left|\alpha_{n}(\mathbf{x})\right|}{N}\right)(\mathrm{dB}), & \\
& n=1,2, \ldots, N-1,
\end{aligned}
$$

TABLE 3: Algorithms comparison for suppressing narrow interference in multistopbands.

\begin{tabular}{lccc}
\hline \multirow{2}{*}{ Algorithms } & \multicolumn{3}{c}{ Multistopbands } \\
& PSP $(\mathrm{dB})$ & ASP $(\mathrm{dB})$ & Execution time (s) \\
\hline SARAP & -21.464 & -13.742 & 0.286 \\
SCAN & -8.373 & -14.614 & 1.027 \\
WeSCAN & -28.214 & -11.804 & 31.040 \\
\hline
\end{tabular}

$$
\mathrm{PSP}=10 \log \left(\max \left|\mathrm{PSD}_{\text {stop }}\right|\right)(\mathrm{dB})
$$

where $\mathrm{PSD}_{\text {stop }}$ denotes the power spectral density (PSD) in stopbands.

From Table 3, one can easily find that WeSCAN has much lower spectrum suppression than SARAP and SCAN, but at the cost of increasing ASP. And WeSCAN, with a largescale FFT operation and eigenvalue decomposition in each iteration, has occupied more time than others. Moreover, SARAP has shown some highlighted performance in the sense of PSP, but it might fall behind SCAN in terms of ASP; meanwhile its execution time is less than others. More specifically, in Figure 3(b), WeSCAN has achieved lower averaged spectrum suppression than SCAN and SARAP, and SARAP possesses lower averaged spectrum magnitude than SCAN, while the sidelobes suppression in Figure 3(a) seems inconspicuous (but from Table 3, we could distinguish this difference). Compared with SCAN and WeSCAN, SARAP incorporates the advantage of stopband suppression and 


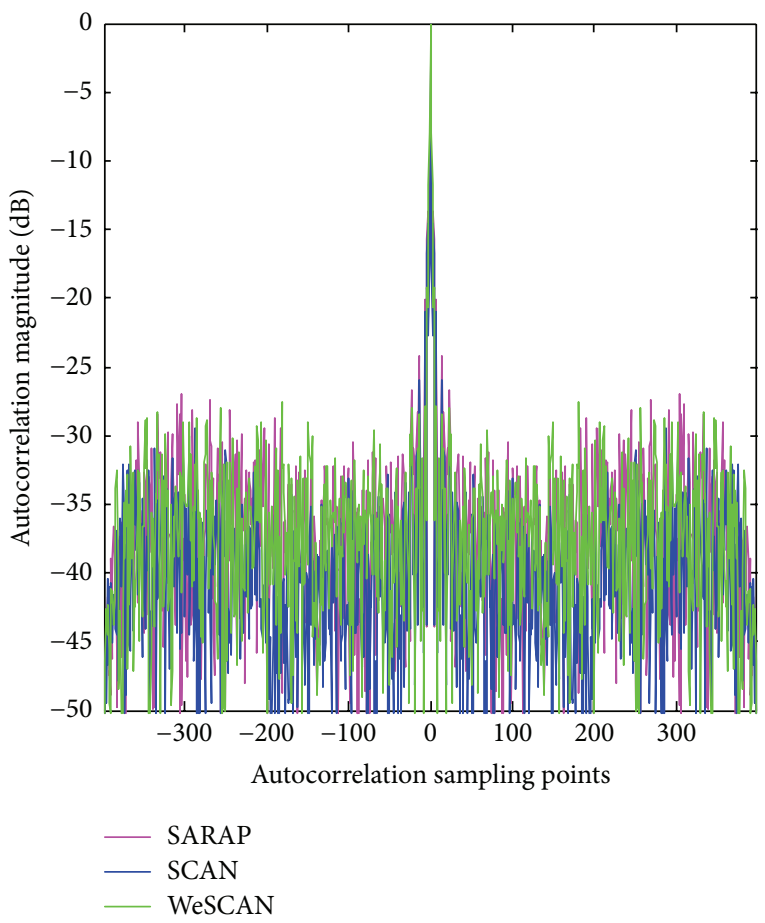

(a)

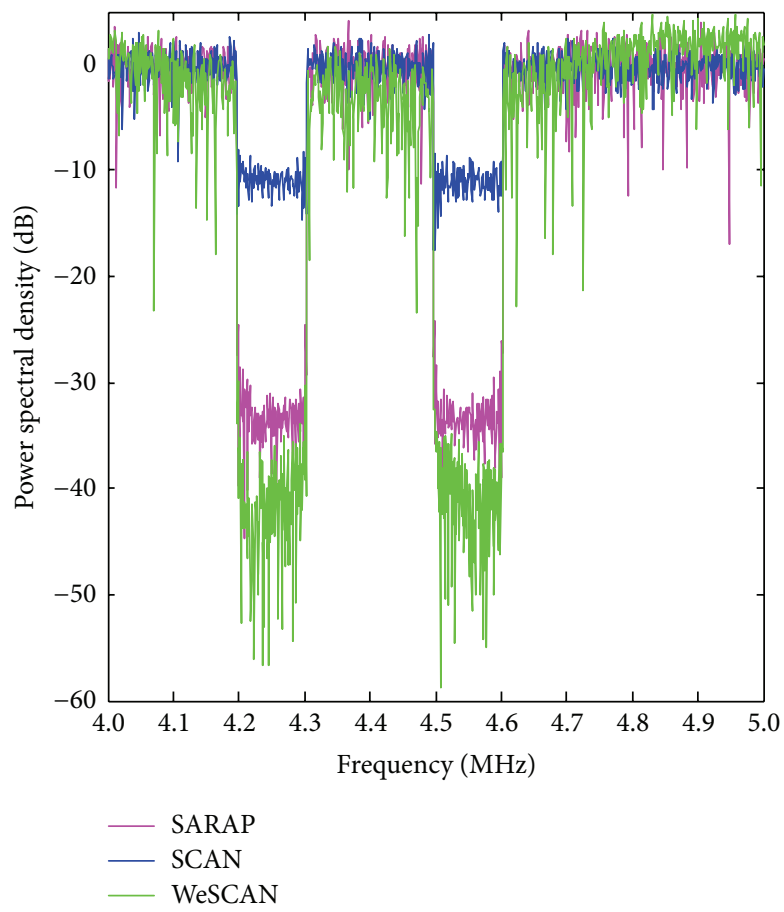

(b)

FIGURE 3: Autocorrelation magnitude and PSD comparison; (a) illustrates range sidelobes suppression; (b) illustrates multistopbands suppression.

TABLE 4: Algorithms comparison for suppressing narrow interference in multistopbands.

\begin{tabular}{lccc}
\hline \multirow{2}{*}{ Algorithms } & \multicolumn{3}{c}{ Multistopbands } \\
& PSP $(\mathrm{dB})$ & ASP $(\mathrm{dB})$ & Execution time (s) \\
\hline SARAP & -21.443 & -14.253 & 0.106 \\
ISAA & -17.035 & -13.174 & 0.415 \\
PAIA & -14.404 & -9.173 & 892.838 \\
\hline
\end{tabular}

execution time together. And we could attribute these traits to the relaxation factor and accelerated factor which exploit local space and more likely approach to the global solution.

Additionally, we propose another scenario and assume that multistopbands locate in $[4.03,4.15] \cup[4.20,4.26] \cup$ $[4.28,4.35] \cup[4.37,4.40] \cup[4.42,4.45] \mathrm{MHz}$; thus passbands would cover the remaining part of $[4.0,4.71] \mathrm{MHz}$. The pulse duration is $400 \mu$ s and sampling rate is $1 \mathrm{MHz}$; thus there are 400 samples. SARAP would be compared with PAIA [8] and ISAA [14] under parameters $\gamma=0.6, K=1000$, and $\varepsilon=10^{-3}$.

It is not difficult to observe that, from Table 4, SARAP and ISAA all outperform PAIA in the sense of PSP, ASP, and execution time. And SARAP has obtained lower spectrum suppression which is consistent with Figure 4(b). As is known PAIA combines cyclic algorithm and derivative-based nonlinear programming together, and its computational complexity is quadratic with respect to the number of samples, while SARAP and ISAA with derivative-free mechanism seem easily implemented. From Figure 4(a), one can find that
TABLE 5: Algorithms comparison for suppressing narrowband interference and sidelobe interference.

\begin{tabular}{lccc}
\hline \multirow{2}{*}{ Algorithms } & \multicolumn{3}{c}{ Multistopbands } \\
& PSP $(\mathrm{dB})$ & ASP $(\mathrm{dB})$ & Execution time $(\mathrm{s})$ \\
\hline SARAP & -24.551 & -12.269 & 0.903 \\
ISAA & -20.017 & -11.441 & 2.013 \\
\hline
\end{tabular}

PAIA undergoes high range sidelobes which would impact on target detection.

4.3. Design Unimodular Waveform with Low Range Sidelobes in Prescribed Intervals and Sparse Frequency Properties as Well. Assume that multistopbands locate in $[4.2,4.3] \cup$ $[4.5,4.6] \mathrm{MHz}$ and passbands would cover the remaining part of $[4,4.71] \mathrm{MHz}$; additionally, some powerful interference lies in intervals $Q_{r}=[20: 80] \cup[200: 300]$. The pulse duration is $500 \mu$ s and sampling rate is $2 \mathrm{MHz}$; thus there are $1000 \mathrm{sam}-$ ples. With identical random initialization, SARAP of Algorithm 3 would be compared with ISAA under parameters $\gamma=0.6, K=1500$, and $\varepsilon=10^{-4}$.

Note that low range sidelobes and sparse frequency properties have made waveform synthesis challengeable. From Table 5, one can find that the performance of SARAP is better than ISAA; for example, SARAP is not only more efficient than ISAA in terms of executing time but also has a better performance of PSP and ASP. Given identical simulation parameters, SARAP has achieved lower suppression in multistopbands and obtained lower range sidelobes in prescribed 


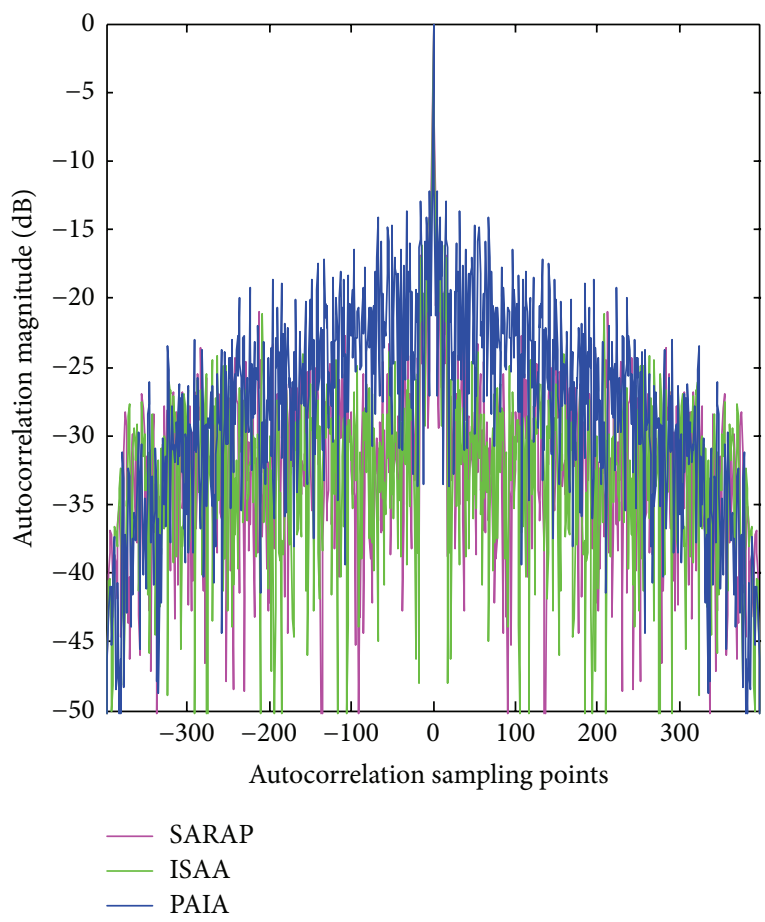

(a)

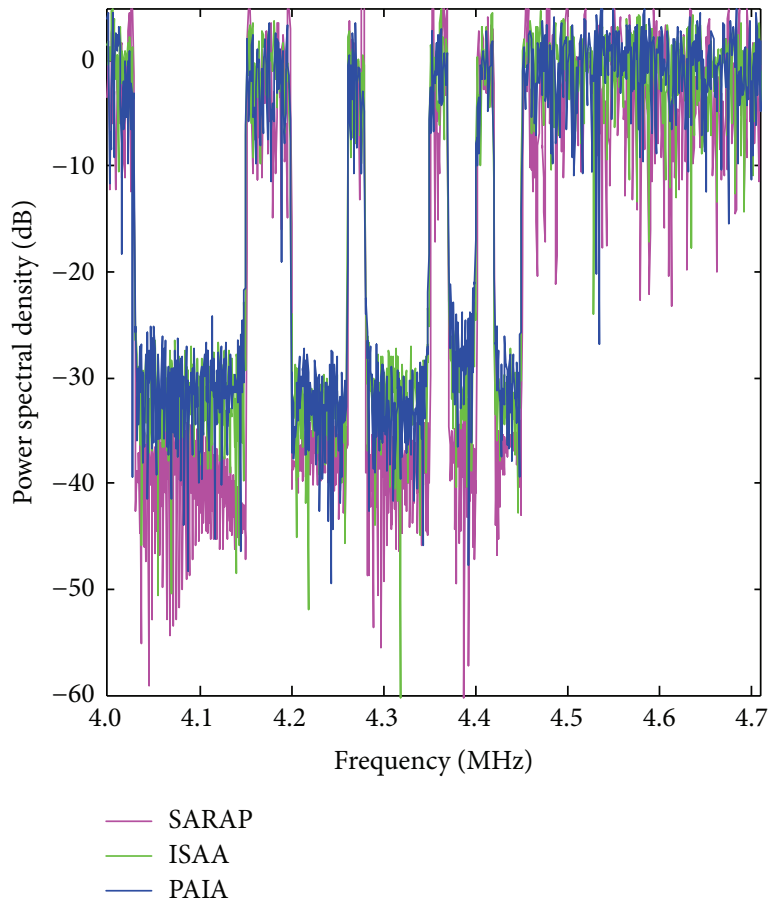

(b)

FIGURE 4: Autocorrelation magnitude and PSD comparison; (a) illustrates range sidelobes suppression; (b) illustrates multistopbands suppression.

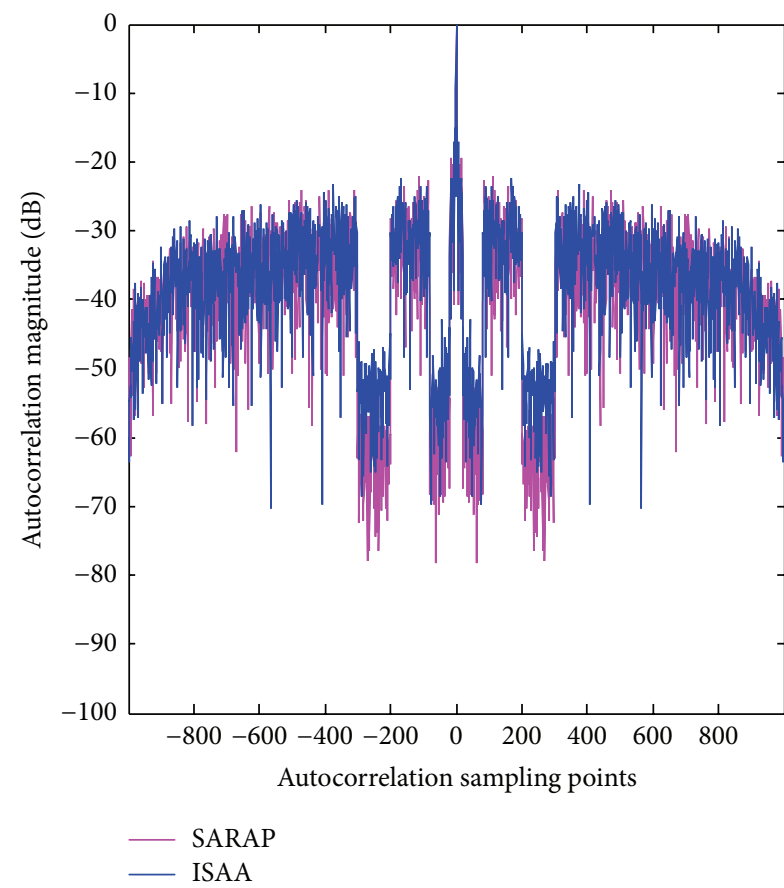

(a)

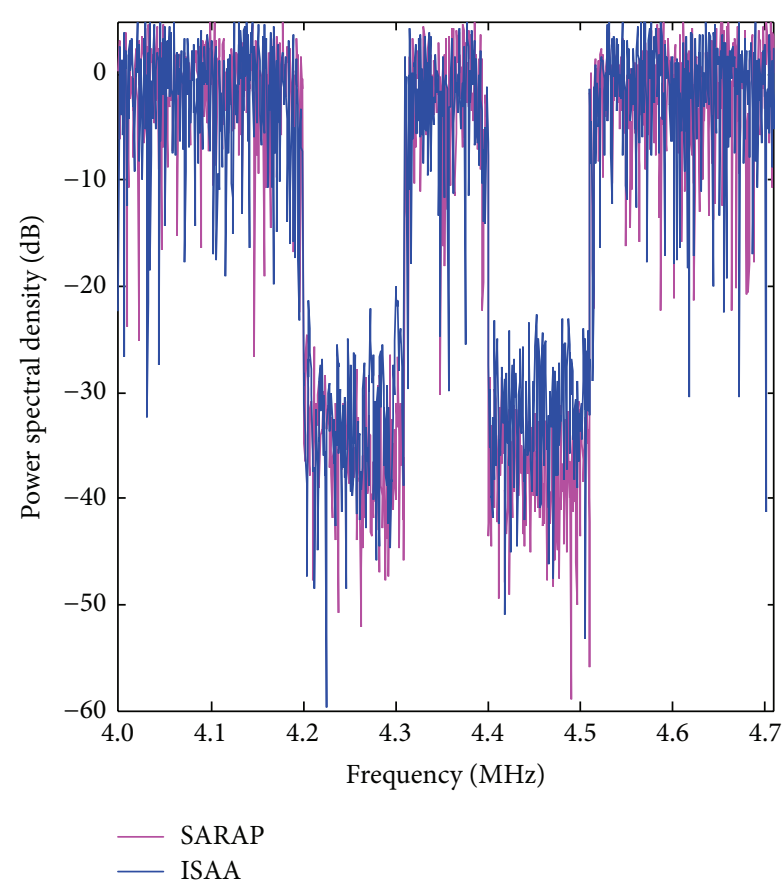

(b)

FIGURE 5: Autocorrelation magnitude and PSD comparison; (a) illustrates range sidelobes suppression; (b) illustrates multistopbands suppression. 
intervals. This phenomenon might attribute to relaxation factor and accelerated factor which could exploit local space and avoid stagnation See Figure 5.

\section{Conclusions}

In this paper, we propose SARAP to design unimodular waveform with sparse frequency and low range sidelobes as well. This method, based on FFT and phase retrieval, has achieved outstanding performance via its relaxation factor and accelerated factor. Simulations have shown that SARAP outperforms ISAA, WeCAN, SCAN, PAIA, and so forth. SARAP could exploit local space and more likely approach to the global solution in the case of random initialization, which would make far-reaching influence on engineering.

\section{Competing Interests}

The authors declare that they have no competing interests.

\section{Acknowledgments}

This work is supported by National Natural Science Foundation of China (61371181).

\section{References}

[1] S. Haykin, "Cognitive radar: a way of the future," IEEE Signal Processing Magazine, vol. 23, no. 1, pp. 30-40, 2006.

[2] A. Aubry, A. De Maio, Y. Huang, M. Piezzo, and A. Farina, "A new radar waveform design algorithm with improved feasibility for spectral coexistence," IEEE Transactions on Aerospace and Electronic Systems, vol. 51, no. 2, pp. 1029-1038, 2015.

[3] C. Mai, S. Lu, J. Sun, and G. Wang, "Codesign of beam pattern and sparse frequency waveforms for MIMO radar," International Journal of Antennas and Propagation, vol. 2015, Article ID 563941, 12 pages, 2015.

[4] A. Aubry, A. De Maio, M. Piezzo, and A. Farina, "Radar waveform design in a spectrally crowded environment via nonconvex quadratic optimization," IEEE Transactions on Aerospace and Electronic Systems, vol. 50, no. 2, pp. 1138-1152, 2014.

[5] G. Wang and Y. Lu, "Sparse frequency waveform design based on PSD fitting," in Proceedings of the 36th IEEE International Conference on Acoustics, Speech, and Signal Processing (ICASSP '11), pp. 2860-2863, IEEE, Prague, Czech Republic, May 2011.

[6] P. Stoica, H. He, and J. Li, "New algorithms for designing unimodular sequences with good correlation properties," IEEE Transactions on Signal Processing, vol. 57, no. 4, pp. 1415-1425, 2009.

[7] H. He, P. Stoica, and J. Li, "Waveform design with stopband and correlation constraints for cognitive radar," in Proceedings of the 2nd International Workshop on Cognitive Information Processing (CIP '10), pp. 344-349, IEEE, Elba, Italy, June 2010.

[8] G. Wang and Y. Lu, "Designing single/multiple sparse frequency waveforms with sidelobe constraint," IET Radar, Sonar \& Navigation, vol. 5, no. 1, pp. 32-38, 2011.

[9] J. Song, P. Babu, and D. P. Palomar, "Optimization methods for designing sequences with low autocorrelation sidelobes," IEEE Transactions on Signal Processing, vol. 63, no. 15, pp. 3998-4009, 2015.
[10] Y.-C. Wang, L. Dong, X. Xue, and K.-C. Yi, "On the design of constant modulus sequences with low correlation sidelobes levels," IEEE Communications Letters, vol. 16, no. 4, pp. 462-465, 2012.

[11] H. Wu, Z. Song, H. Fan, Y. Li, and Q. Fu, "A new algorithm for sparse frequency waveform design with range sidelobes constraint," Chinese Journal of Electronics, vol. 24, no. 3, pp. 604610, 2015

[12] R. Kassab, M. Lesturgie, and J. Fiorina, "Alternate projections technique for radar waveform design," in Proceedings of the International Radar Conference 'Surveillance for a Safer World' (RADAR '09), IEEE, Bordeaux, France, December 2009.

[13] Y.-N. Zhao, F.-C. Li, T. Zhang, and Z.-Q. Zhou, "Computational design of optimal waveforms for MIMO radar via multi-dimensional iterative spectral approximation," Multidimensional Systems and Signal Processing, vol. 27, no. 1, pp. 43-60, 2016.

[14] F.-C. Li, Y.-N. Zhao, and X.-L. Qiao, "A waveform design method for suppressing range sidelobes in desired intervals," Signal Processing, vol. 96, pp. 203-211, 2014.

[15] S. Wang, "Efficient heuristic method of search for binary sequences with good aperiodic autocorrelations," Electronics Letters, vol. 44, no. 12, pp. 731-732, 2008.

[16] S. Ahmed, J. S. Thompson, Y. R. Petillot, and B. Mulgrew, "Finite alphabet constant-envelope waveform design for MIMO radar," IEEE Transactions on Signal Processing, vol. 59, no. 11, pp. 53265337, 2011.

[17] A. Aubry, A. De Maio, B. Jiang, and S. Zhang, "Ambiguity function shaping for cognitive radar via complex quartic optimization," IEEE Transactions on Signal Processing, vol. 61, no. 22, pp. 5603-5619, 2013.

[18] V. V. Zhuk, "Approximation of functions in the space $C(\mathbb{R})$. Parseval-type equality," Voprosy Mekhaniki i Protsessov Upravleniya, vol. 16, pp. 105-118, 1995.

[19] L. K. Patton and B. D. Rigling, "Phase retrieval for radar waveform optimization," IEEE Transactions on Aerospace and Electronic Systems, vol. 48, no. 4, pp. 3287-3302, 2012.

[20] J. A. Tropp, I. S. Dhillon, R. W. Heath Jr., and T. Strohmer, "Designing structured tight frames via an alternating projection method," IEEE Transactions on Information Theory, vol. 51, no. 1, pp. 188-209, 2005.

[21] R. Escalante and M. Raydan, Alternating Projection Methods, SIAM, 2011.

[22] H. S. Hundal, "An alternating projection that does not converge in norm," Nonlinear Analysis: Theory, Methods \& Applications, vol. 57, no. 1, pp. 35-61, 2004.

[23] A. Cegielski and A. Suchocka, "Relaxed alternating projection methods," SIAM Journal on Optimization, vol. 19, no. 3, pp. 1093-1106, 2008.

[24] S. Pillai, K. Li Y, I. Selesnick et al., Waveform Diversity: Theory \& Applications: Theory \& Application, McGraw Hill Professional, New York, NY, USA, 2011.

[25] H. W. Leong and B. Dawe, Channel Availability for East Coast High Frequency Surface Wave Radar Systems, Defence Research Establishment, Ottawa, Canada, 2001. 


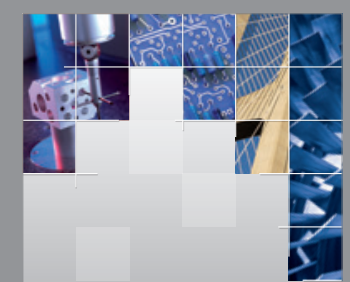

\section{Enfincering}
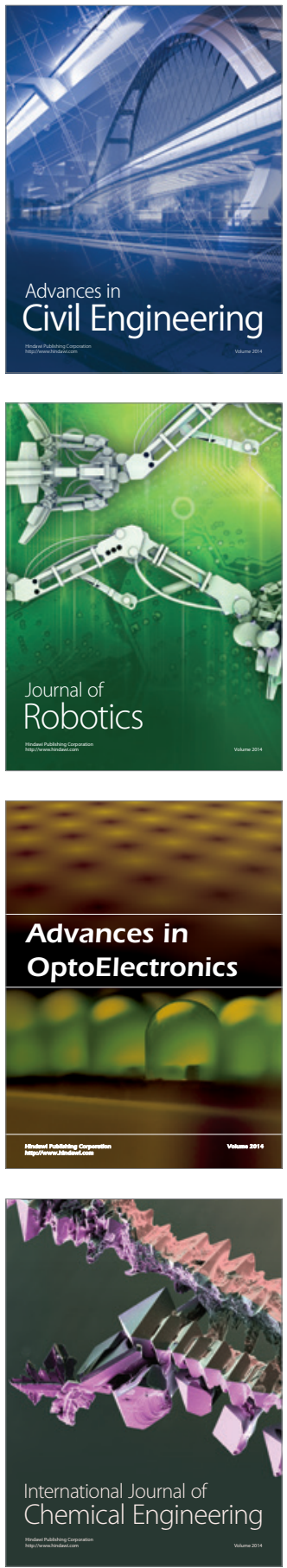

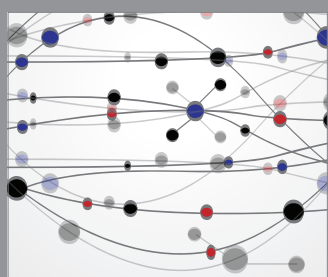

The Scientific World Journal

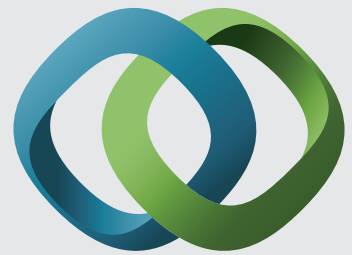

\section{Hindawi}

Submit your manuscripts at

http://www.hindawi.com
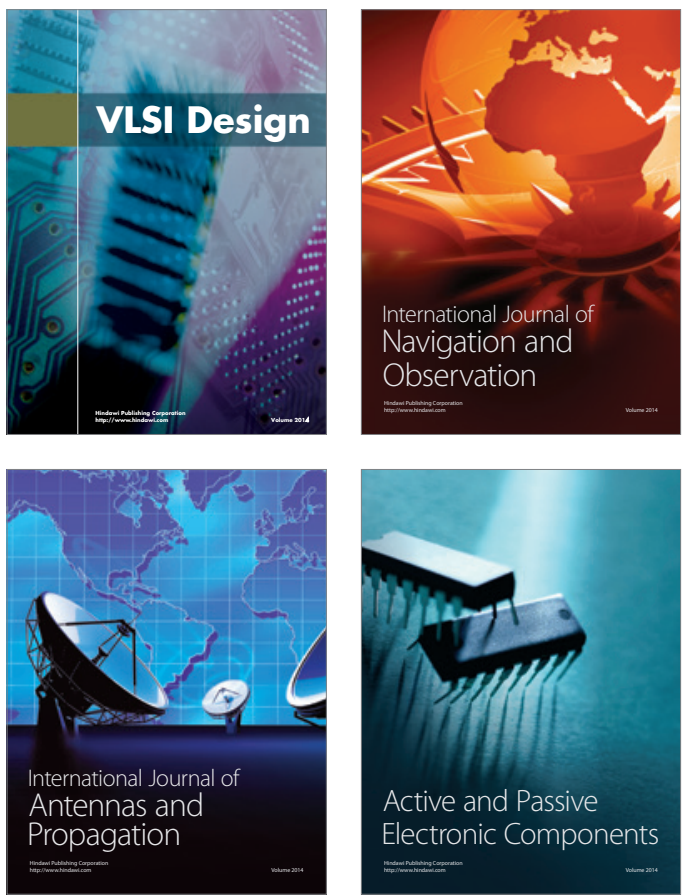
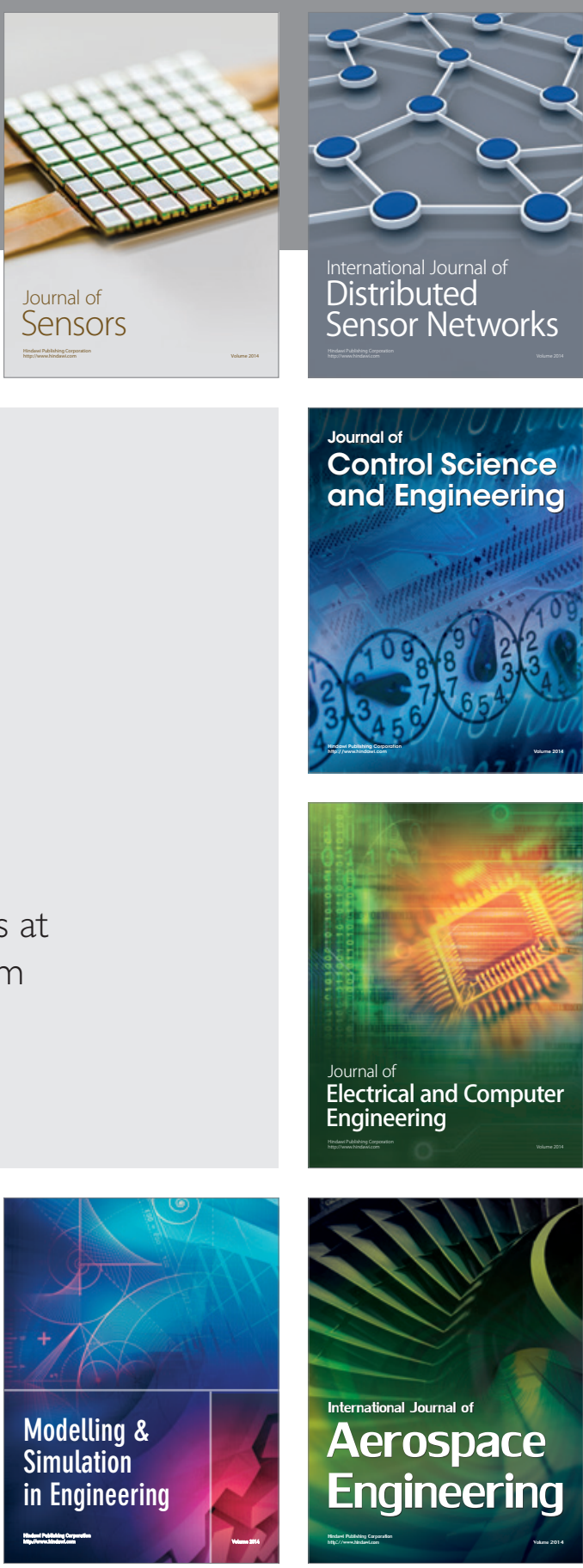

International Journal of

Distributed

Sensor Networks

Journal of

Control Science

and Engineering
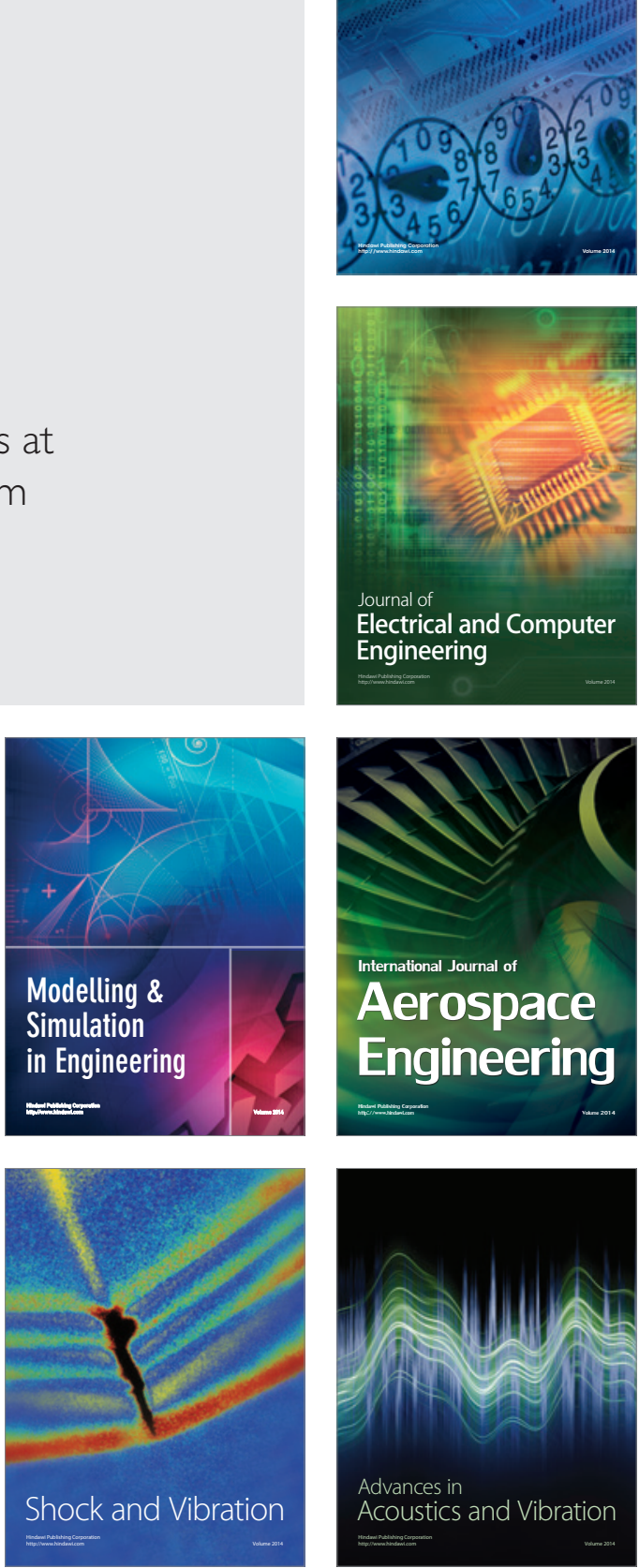